\title{
Chloroplast ribonucleoprotein-like proteins of the moss Physcomitrella patens are not involved in RNA stability and RNA editing
}

\author{
H. UCHIYAMA*, M. ICHINOSE*,**, and M. SUGITA*,+, \\ Center for Gene Research, Nagoya University, Chikusa-ku, Nagoya 464-8602, Japan* \\ Institute of Transformative Bio-Molecules, Nagoya University, Chikusa-ku, Nagoya 464-8602, Japan ${ }^{* *}$
}

\begin{abstract}
Many RNA recognition motif (RRM)-containing proteins are known to exist in chloroplasts. Major members of the RRM protein family, which are chloroplast ribonucleoproteins (cpRNPs), have been investigated in seed plants, including tobacco and Arabidopsis thaliana, but never in early land plants, such as bryophytes. In this study, we surveyed RRM proteins encoded in the moss Physcomitrella patens genome and predicted 25 putative chloroplast RRM proteins. Among them, two RRM-containing proteins, PpRBP2a and PpRBP2b, resembled cpRNPs and were thus referred to as cpRNPlike proteins. However, knockout mutants of either one or two $P p R B P 2$ genes exhibited a wild type-like phenotype. Unlike Arabidopsis cpRNPs, the levels of mRNA accumulation in chloroplasts were not affected in the PpRBP2 knockout mutants. In addition, the efficiency of RNA editing was also not altered in the mutants. This suggests that PpRBP2a and b may be functionally distinct from Arabidopsis cpRNPs.
\end{abstract}

Additional key words: pentatricopeptide repeat protein; photosynthesis gene; posttranscriptional regulation; RNA-binding protein; RNA processing.

The components of the photosynthetic apparatus, as well as the transcriptional and translational machinery, are encoded by the chloroplast and nuclear genomes in algae and plants (Allen et al. 2011). The nucleus-encoded components are synthesized as precursor polypeptides that are imported into the chloroplasts and subsequently assembled with chloroplast-encoded components. The expression of many chloroplast genes is posttranscriptionally regulated by nuclear-encoded RNA binding proteins (del Campo 2009, Stern et al. 2010). Among them, RNA recognition motif (RRM)-containing proteins are considered to be major regulators in various types of RNA metabolism in chloroplasts (Nickelsen 2003, Maris et al. 2005, Tillich et al. 2010). The RRM is about 80 amino acids long and contains two consensus motifs, RNP1 and RNP2. RNP1 is highly conserved with 8 amino acids located near its center, while RNP2 is not as well conserved with 6 amino acids found near its N-terminus. RRM folds into a $\beta 1-\alpha 1-\beta 2-\beta 3-\alpha 2-\beta 4$ structure and forms an anti-parallel four-stranded $\beta$-sheet, $\beta 2 \beta 3 \beta 1 \beta 4$, in this order. RNP1 and RNP2 are juxtaposed on the two central $\beta$ strands, $\beta 3$ and $\beta 1$, respectively (Nagai et al. 1990). This arrangement is responsible for RNA binding.

Chloroplast RRM proteins were first identified in tobacco as approximately $30-\mathrm{kDa}$ single-stranded DNAbinding proteins (Li and Sugiura 1990). They were named 'chloroplast ribonucleoprotein' (cpRNP) as they featured a high abundance of chloroplasts and their amino acid sequences were similar to yeast and animal RRMcontaining ribonucleoproteins, such as heterogeneous nuclear ribonucleoprotein (hnRNP) and U1 small nuclear ribonucleoprotein (snRNP). Nuclear-encoded cpRNPs are 250-340 amino acids long and have a characteristic domain organization: an N-terminal transit peptide, which is removed after import into chloroplasts, is followed by an acidic region at the N-terminus of the mature cpRNP and two consecutive RRMs. The cpRNPs, which associate with ribosome-free mRNAs and pre-tRNAs and form

Received 29 April 2017, accepted 17 July 2017, published as online-first 26 September 2017.

${ }^{+}$Corresponding author; e-mail: sugita@gene.nagoya-u.ac.jp

Abbreviations: 5-CLF - 5-formyltetrahydrofolate cyclo-ligase; cpRNP - chloroplast ribonucleoprotein; GFP - green fluorescence protein; GUS - B-glucuronidase; hnRNP - heterogeneous nuclear ribonucleoprotein; KO - knockout; PABP - poly(A)-binding protein; PPR - pentatricoepetide repeat; RRM - RNA recognition motif; snRNP - small nuclear ribonucleoprotein.

Acknowledgements: We thank Professor Julian Eaton-Rye for inviting us to join in the Photosynthetica special issue to celebrate Govindjee's 85 th birthday. This work was supported by JSPS KAKENHI Grant Numbers 15K14917, 17K08195 (to MS). 
heterogeneous complexes in the stroma (Nakamura et al. 1999), are involved in mRNA stabilization (Nakamura et al. 2001), mRNA 3'-end formation (Schuster and Gruissem 1991), and RNA editing (Hirose and Sugiura 2001, Tillich et al. 2009). Arabidopsis thaliana has 10 members of the cpRNP family. In addition to $10 \mathrm{cpRNPs}$, at least 13 non-cpRNP RRM proteins have been identified in A. thaliana, but their function in chloroplasts remains unknown (Ruwe et al. 2011). It is possible that cpRNPs and non-cpRNP RRM proteins are widely distributed among land plant species, including early land plants, such as bryophytes.

To identify chloroplast RRM proteins in the moss $P$. patens, we surveyed the $P$. patens genome for RRM proteins that were predicted to be localized in chloroplasts. To this end, various RRMs of several RRM proteins were used as queries and BLAST searched against the plant genomics resource Phytozome v. 11.0 (https://phytozome. jgi.doe.gov/pz/portal.html). The localization of chloroplasts was predicted by TargetP and Predotar programs. This analysis revealed over $220 \mathrm{RRM}$ proteins in $P$. patens, $11 \%$ of which were predicted to be chloroplast RRM proteins.
Two chloroplast pentatricopeptide repeat (PPR) proteins, PpPPR_3 (Pp3c5_10110) and PpPPR_76 (Pp3c16 280), which consist of an N-terminal RRM and 15 PPR motifs, were previously identified in $P$. patens (Sugita et al. 2013). These two PPR proteins show 59\% amino acid identity and are $87 \%$ similar to each other. In this study, we found 25 additional chloroplastic RRM proteins, including their paralogs (Table 1). These could be further divided into several groups based on their amino acid length and identities, position of the RRM, and the presence of additional domains (Fig. 1S, supplement available online). Pp3c9_17530 (named PpRBP1) is 674 amino acids long and contains a C-terminal RRM. This seems to be unique to $P$. patens because of its absence in Arabidopsis. Pp3c25 3310 contains a C-terminal PWI domain (Pfam ID: PF01480), which is commonly found in splicing factors. Arabidopsis has two proteins with a PWI domain (At2g29210 and At1g60200). Pp3c25 3310 is likely the homolog of At1g60200. Pp3c18 17520 consists of a domain of the 5-formyltetrahydrofolate cyclo-ligase (5-FCL) family (Pfam ID: PF01812) and a C-terminal RRM. 5-FCL, which catalyzes the interchange of

Table 1. Putative chloroplastic RRM proteins in Physcomitella patens. ${ }^{\text {a }}$ Locus name of RRM protein represents the gene model (version 3.3) assigned in COSMOSS The Physcomitrella patens resource database (www.cosmoss.org). ${ }^{\text {b }}$ Length (aa) includes a transit peptide

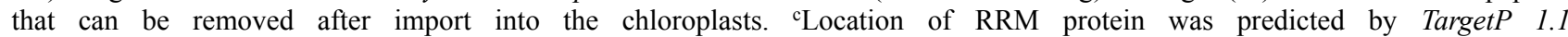
(http://www.cbs.dtu.dk/services/TargetP/) and Predotar (http://urgi.versailles.inra.fr/predotar/predotar.html). Prediction scores by Target $P$ are indicated in parenthesis. Reliability class, from 1 (score, $>0.8), 2(0.8-0.6), 3(0.6-0.4)$, and $4(0.4-0.2)$, where $\mathrm{C} 1$ indicates the strongest chloroplast prediction. $\mathrm{C}, \mathrm{M}, \mathrm{N}$, and n.d. indicate chloroplasts, mitochondria, nucleus, and 'not determined', respectively. d According to Mueller et al. (2014), Fesenko et al. (2016).

\begin{tabular}{|c|c|c|c|c|c|c|c|c|}
\hline $\begin{array}{l}\text { Locus name }{ }^{\mathrm{a}} \\
\text { (Paralogous loci) }\end{array}$ & $\begin{array}{l}\text { RRM } \\
\text { protein name }\end{array}$ & $\begin{array}{l}\text { RRM } \\
\text { No. }\end{array}$ & $\begin{array}{l}\text { Length }^{\mathrm{b}} \\
\text { (aa) }\end{array}$ & $\begin{array}{l}\text { Prodicted } 1 \\
\text { TargetP I }\end{array}$ & $\begin{array}{l}\text { location }^{\mathrm{c}} \\
\text { Predotor }\end{array}$ & $\begin{array}{l}\text { Location } \\
\text { experimentally } \\
\text { determined }\end{array}$ & $\begin{array}{l}\text { Detected in } \\
\text { chloroplasts }^{\mathrm{d}}\end{array}$ & Arabidopsis homologs \\
\hline Pp3c9_17530 & PpRBP1 & 1 & 674 & C1 (0.83) & & $\mathrm{C}$ & & \\
\hline $\begin{array}{l}\text { Pp3c18_16140 } \\
\text { (Pp3c19_7020, } \\
\text { Pp3c1_27480, } \\
\text { Pp3c21_4170) }\end{array}$ & & 1 & 275 & C1 $(0.91)$ & & $\mathrm{N}$ & & At4g35785 \\
\hline $\begin{array}{l}\text { Pp3c12_22790 } \\
\text { (Pp3c8_3970, } \\
\text { Pp3c3_28550 }\end{array}$ & & 1 & 399 & C1 (0.92) & $\mathrm{C}$ & $\mathrm{N}$ & & Atlg16610 (SR45) \\
\hline Pp3c25_3310 & & 1 & 817 & C3 (0.52) & & n.d. & & At1g60200 \\
\hline $\begin{array}{l}\text { Pp3s152_10 } \\
\text { (Pp3s5_15600) }\end{array}$ & SCL30A-like & 1 & 370 & $\mathrm{C} 2(0.61)$ & & $\mathrm{C}$ & & At3g13570 (SCL30A) \\
\hline Pp3c8_3240 & & 1 & 267 & C2 (0.53) & & $\mathrm{N}$ & & At5g04600 \\
\hline Pp3c18_17520 & COG0212-like & 1 & 456 & C2 (0.65) & $\mathrm{C}$ & $\mathrm{C}$ & & Atlg76730 (COG0212) \\
\hline Pp3c1_-̄ 1900 & RBD1-like & 1 & 523 & $\mathrm{C} 1(0.88)$ & $\mathrm{C}$ & $\mathrm{C}$ & & At1g70200 (RBD1) \\
\hline Pp3c1_20440 & & 1 & 561 & C1 (0.89) & $\mathrm{C}$ & n.d. & & At3g27700 \\
\hline Pp3c12 15180 & & 1 & 533 & C4 (0.35) & $\mathrm{C}$ & n.d. & & \\
\hline Pp3c12_9890 & PpRBP2a & 2 & 287 & $\mathrm{C} 1(0.87)$ & $\mathrm{C}$ & $\mathrm{C}$ & Yes & cpRNPs \\
\hline Pp3c24_17170 & PpRBP2b & 2 & 281 & $\mathrm{C} 1(0.91)$ & $\mathrm{C}$ & $\mathrm{C}$ & & cpRNPs \\
\hline Pp3c5_17010 & & 2 & 475 & $\mathrm{C} 2(0.61)$ & $\mathrm{C}$ & n.d. & & At1g09230 (U11/U12-65K) \\
\hline Pp3c8_16740 & PpRBP3a & 3 & 418 & $\mathrm{C} 1(0.84)$ & $\mathrm{C}$ & $\mathrm{C}$ & Yes & \\
\hline Pp3c24_17150 & PpRBP3b & 3 & 478 & M3 $(0.41)$ & & $\mathrm{C}$ and $\mathrm{M}$ & Yes & \\
\hline $\begin{array}{l}\text { Pp3c17_14650 } \\
\left(\mathrm{Pp} 3 \mathrm{c} 14 \_9430\right)\end{array}$ & & 3 & 446 & $\mathrm{C} 3(0.51)$ & $\mathrm{C}$ & $\mathrm{N}$ & & \\
\hline $\begin{array}{l}\text { Pp3c10_4970 } \\
\left(\mathrm{Pp} 3 \mathrm{c} 14 \_4340\right)\end{array}$ & PABP-like & 4 & 643 & C3 $(0.47)$ & & $\mathrm{N}$ & & At2g36660 (PAB7) \\
\hline
\end{tabular}


5-formyltetrahydrofolate to 5,10-methenyltetrahydrofolate, is likely a homolog of Arabidopsis COG0212 that is targeted to chloroplasts and is required for embryo viability (Pribat et al. 2011). However, Arabidopsis COG0212 lacks an RRM. Recently, chloroplast RNAbinding protein RBD1 (At1g70200) and organelle RRM protein 1 (ORRM1, At3g2093) have been characterized in Arabidopsis: RBD1 is involved in chloroplast 23S rRNA processing (Wang et al. 2016), while ORRM1 is the chloroplast RNA editing factor required for several editing sites (Sun et al. 2013). P. patens has a homolog of RBD1 (Pp3c1_41900), but does not possess ORRM1.

Since two RRM-containing proteins, Pp3c12_9890 and Pp3c24_17170, showed 53\% amino acid identity with each other, we named them PpRBP2a and PpRBP2b, respectively. Proteomic analysis detected PpRBP2a in chloroplasts as a highly abundant protein (Mueller et al. 2014, Fesenko et al. 2016). Expression data deposited in Phytozome v. 11.0 indicates that PpRBP2b mRNA is highly expressed. Two PpRBP2 proteins showed high amino acid identity ( $>40 \%$ ) with Arabidopsis cpRNPs, CP28A, CP29B, and CP31B, and may thus be cpRNP-like proteins. The other member containing two RRMs, Pp3c5 17010 is expressed at low levels. Proteins containing three RRMs, Pp3c8_16740 (PpRBP3a) and Pp3c24 17150 (PpRBP3b), were detected in the P. patens chloroplasts by proteomics analyses (Mueller et al. 2014, Fesenko et al. 2016), although their homologs are not found in Arabidopsis. Rather, At2g42890 (AML2, ARABIDOPSIS-MEI12-LIKE 2) was identified in Arabidopsis as a chloroplast protein containing three RRMs (Ruwe et al. 2011), but this protein was not found in $P$. patens. Two poly(A)-binding protein (PABP)-like proteins were identified as chloroplastic RRM proteins. Arabidopsis has eight PABPs, two of which, PAB4 (At2g23350) and PAB7 (At2g36660), were predicted to be chloroplast PABPs (Belostotsky 2003). However, $P$. patens PABP-like proteins were targeted to the nucleus but not to chloroplasts (data not shown).

To confirm the localization of chloroplasts, N-terminal sequences of RRM proteins were fused to green fluorescence protein (GFP) or $\beta$-glucuronidase (GUS) and then expressed in $P$. patens protonema cells (Ichinose et al. 2013). Among the 13 RRM proteins tested, eight were targeted to chloroplasts and five to the nucleus (Table 1; Fig. 2S, supplement available online). It is interesting to observe that PpRBP3b was targeted to both chloroplasts and mitochondria. Thus, at least 10 RRM proteins, including PPR proteins, have been confirmed to exist in $P$. patens chloroplasts. Cellular localization of the remaining RRM proteins has yet to be determined.

Proteomics analysis revealed that some cpRNPs (CP29, CP31, and CP33) were cold-inducible proteins in Arabidopsis and wheat (Amme et al. 2006, Sarhadi et al. 2010). To examine the effect of cold temperature on the expression of five $P p R B P$ genes, moss protonemata grown at $25^{\circ} \mathrm{C}$ were transferred to $4^{\circ} \mathrm{C}$ and grown for $4 \mathrm{~d}$, and

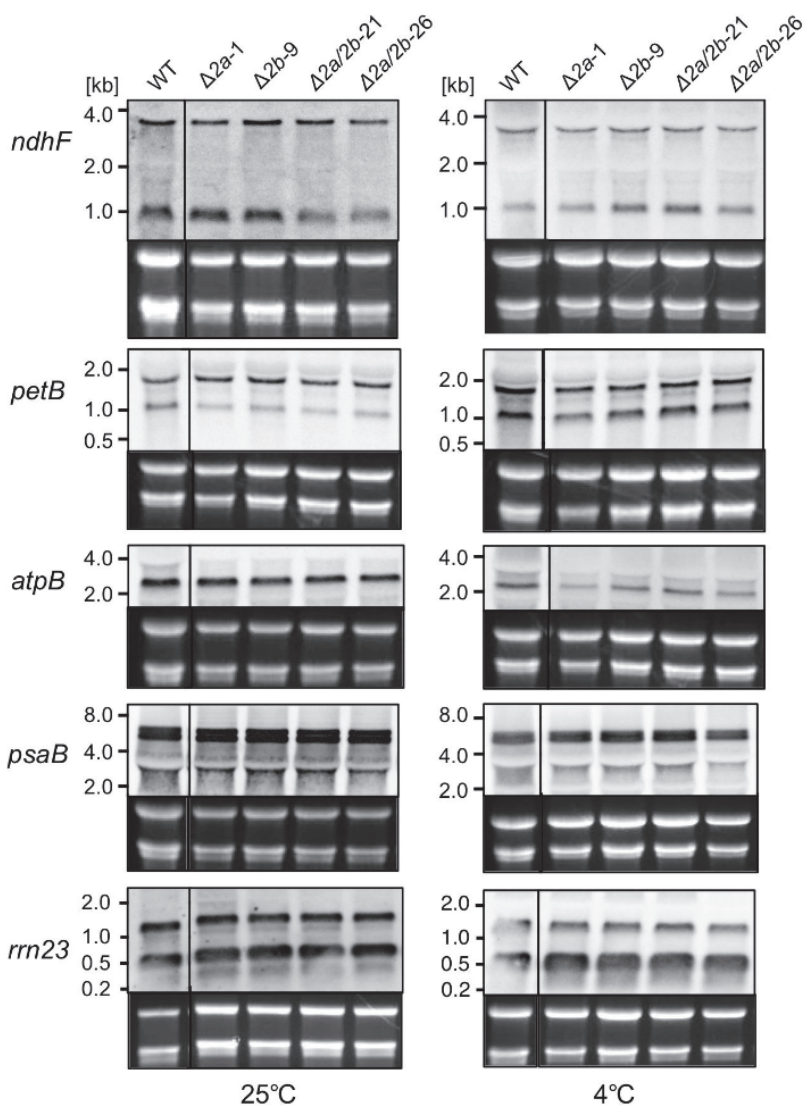

Fig. 1. Northern blot analysis of chloroplast genes. Total RNA from wild type (WT) and knockout $(\mathrm{KO})$ mutant mosses grown at $25^{\circ} \mathrm{C}$ or $4^{\circ} \mathrm{C}$ was separated on a $1 \%$ agarose gel and subjected to Northern blot analysis using chloroplast gene-specific DNA probes (Goto et al. 2016). Analyzed mutants indicate single $(\Delta 2 a-1, \Delta 2 b-9)$ or double $(\Delta 2 a / 2 b-21, \Delta 2 a / 2 b-26)$ mutants of PpRBP2 genes. Ten $\mu \mathrm{g}$ of total RNA was loaded on the gel to detect mRNAs (5 $\mu \mathrm{g}$ of total RNA to detect processed $23 \mathrm{~S}$ rRNAs). The positions of RNA size markers are indicated as 0.2 to $8 \mathrm{~kb}$.

quantitative RT-PCR was performed on these genes. The transcript levels of $P p R B P 2 a$ and $P p R B P 2 b$ increased 3and 6-fold, respectively, at $4^{\circ} \mathrm{C}$ compared to levels at $25^{\circ} \mathrm{C}$. In contrast, $P p R P B 1, P p R B P 3 a$ and $P p R B P 3 b$ transcripts were detected at similar levels in moss grown at 25 and $4{ }^{\circ} \mathrm{C}$. This suggests that PpRBP $2 \mathrm{a}$ and PpRBP $2 b$ can be classified as cpRNPs. To further investigate their function, we constructed single and double knockout (KO) mutants of $P p R B P 2$ genes. Null KO mutants were verified by RT-PCR using gene-specific primers (Fig. $3 \mathrm{~S} A$, supplement available online). Surprisingly, all $\mathrm{KO}$ mutants exhibited the wild type (WT)-like phenotype, even on different growth media (BCD or BCDATG) and at $25^{\circ} \mathrm{C}$ or at $4^{\circ} \mathrm{C}$ (Fig. $3 \mathrm{SB}$, supplement available online). Single and double mutants of Arabidopsis CP31A and CP29A exhibited the WT-like phenotype at $23^{\circ} \mathrm{C}$, while the cp29a single and cp29a/31a double mutants showed bleaching of newly emerged leaves at $8^{\circ} \mathrm{C}$ (Kupsch et al. 2012). The loss of Arabidopsis cpRNP CP33A yielded 
albino seedlings that exhibited aberrant leaf development (Teubner et al. 2017). A global loss of the accumulation and processing of chloroplast transcripts was observed in cp33a null mutants (Teubner et al. 2017). Similarly, CP31A and CP29A are required for the accumulation of target mRNAs and for processing by a hidden break in $23 \mathrm{~S}$ rRNA under cold stress (Kupsch et al. 2012). They are thought to confer cold stress tolerance by influencing various chloroplast RNA processing steps (Kupsch et al. 2012). In contrast, no single and double KO mutants of $P p R B P 2$ genes affected the levels of accumulation of the examined $n d h F$, pet $B, a t p B$, and $p s a B$, and processed $23 \mathrm{~S}$ rRNA transcripts (Fig. 1).

Tobacco cpRNP CP31 was shown by an in vitro assay to play a role in C-to-U RNA editing of $p s b L$ and $n d h B$ transcripts as a common editing factor (Hirose and Sugiura 2001). In Arabidopsis, the loss of CP31A influences the efficiency of editing at 13 out of 34 examined editing sties in chloroplasts (Tillich et al. 2009). In this mutant, the efficiency of RNA editing at an editing site in $n d h F$ and at three sites in $n d h D$ transcripts was reduced to $60 \%$ of the level in WT transcripts (Tillich et al. 2009). In P. patens (moss) chloroplasts, two editing sites have been identified in chloroplasts (Miyata and Sugita 2004). Editing at the rps 14-C2 site occurs at 70-80\% efficiency and creates a translation initiation codon AUG. In addition, the rps 14$1 \mathrm{C}$ site in the 5'-untranslated region of the rps 14 transcript is edited at a very low efficiency $(<10 \%)$. This editing is

\section{References}

Allen J.F., de Paula W.B.M., Puthiyaveetil S. et al.: A structural phylogenetic map for chloroplast photosynthesis. - Trends Plant Sci. 16: 645-655, 2011.

Amme S., Matros A., Schlesier B. et al.: Proteome analysis of cold stress response in Arabidopsis thaliana using DIGEtechnology. - J. Exp. Bot. 57: 1537-1546, 2006.

Belostotsky D.A.: Unexpected complexity of poly(A)-binding protein gene families in flowering plants: Three conserved lineages that are at least 200 million years old and possible auto- and cross-regulation. - Genetics 163: 311-319, 2003.

del Campo E.M.: Post-transcriptional control of chloroplast gene expression. - Gene Reg. Syst. Biol. 3: 31-47, 2009.

Fesenko I., Seredina A., Arapidi G. et al.: The Physcomitrella patens chloroplast proteome changes in response to protoplastation. - Front. Plant Sci. 7: 1661, 2016.

Goto S., Kawaguchi Y., Sugita C. et al:: P-class pentatricopeptide repeat protein PTSF1 is required for splicing of the plastid pretRNA ${ }^{\text {Ile }}$ in Physcomitrella patens. - Plant J. 86: 493-503, 2016.

Hirose T., Sugiura M.: Involvement of a site-specific trans-acting factor and a common RNA-binding protein in the editing of chloroplast mRNAs: development of a chloroplast in vitro RNA editing system. - EMBO J. 20: 1144-1152, 2001.

Ichinose M., Sugita C., Yagi Y. et al.: Two DYW subclass PPR proteins are involved in RNA editing of $c c m F c$ and atp9 transcripts in the moss Physcomitrella patens: First complete set of PPR editing factors in plant mitochondria. - Plant Cell Physiol. 54: 1907-1916, 2013.

Kupsch C., Ruwe H., Gusewski S. et al.: Arabidopsis chloroplast unique to $P$. patens and has not been found in other plant species (Miyata and Sugita 2004). To investigate the effect of RNA editing at these sites in the KO mutants of $P p R B P 2$ genes, we cloned and sequenced 20 rps 14 cDNAs. This analysis showed that the efficiency of RNA editing at the rps 14-C2 site was $65 \%$ in WT and $75 \%$ in the PpRBP $2 a / 2 b$ double KO mutant. The efficiency of RNA editing at the rps $14-1 \mathrm{C}$ site was $10 \%$ in WT and $5 \%$ in the double KO mutant. This suggests that cpRNP-like PpRBP2a and PpRBP2b might not be primarily involved in RNA editing.

Our results showed that various types of RRM proteins exist in the moss $P$. patens chloroplasts. Half of the members of the chloroplast RRM family are cpRNPs in Arabidopsis (Ruwe et al. 2011), while only two cpRNPlike RRM proteins exist in $P$. patens. The $P$. patens cpRNP-like proteins, PpRBP2a and PpRBP2b, are structurally similar to Arabidopsis cpRNPs, especially CP28, CP29, and CP31, but their function does not seem to be identical. Chloroplast RRM-type RNA-binding proteins might have expanded during the evolution of plants. The RRM protein family is thought to govern chloroplast gene expression by regulating RNA metabolism. To understand the specialized posttranscriptional mechanism in chloroplasts, functional characterization of RRM proteins and other related RNA-binding proteins needs to be urgently performed.

RNA binding proteins CP31A and CP29A associate with large transcript pools and confer cold stress tolerance by influencing multiple chloroplast RNA processing steps. - Plant Cell 24: 4266-4280, 2012.

Li Y., Sugiura M.: Three distinct ribonucleoproteins from tobacco chloroplasts: each contains a unique amino terminal acidic domain and two ribonucleoprotein consensus motifs. EMBO J. 9: 3059-3066, 1990.

Maris C., Dominguez C., Allain F.H.T.: The RNA recognition motif, a plastic RNA-binding platform to regulate posttranscriptional gene expression. - FEBS J. 272: 2118-2131, 2005.

Miyata Y., Sugita M.: Tissue- and stage-specific RNA editing of rps 14 transcripts in moss (Physcomitrella patens) chloroplasts. - J. Plant Physiol. 161: 113-115, 2004.

Mueller S.J., Lang D., Hoernstein S.N.W. et al.: Quantitative analysis of the mitochondrial and plastid proteomes of the moss Physcomitrella patens reveals protein macrocompartmentation and microcompartmentation. - Plant Physiol. 164: 2081-2095, 2014.

Nagai K., Oubridge C., Jessen T.H. et al.: Crystal structure of the RNA-binding domain of the U1 small nuclear ribonucleoprotein A. - Nature 348: 515-520, 1990.

Nakamura T., Ohta M., Sugiura M. et al:: Chloroplast ribonucleoproteins are associated with both mRNAs and intron-containing precursor tRNAs. - FEBS Lett. 460: 437$441,1999$.

Nakamura T., Ohta M., Sugiura M. et al:: Chloroplast 


\section{H. UCHIYAMA et al.}

ribonucleoproteins function as a stabilizing factor of ribosomefree mRNAs in the stroma. - J. Biol. Chem. 276: 147-152, 2001.

Nickelsen J.: Chloroplast RNA-binding proteins. - Curr. Genet. 43: 392-399, 2003.

Pribat A., Blaby I.K., Lara-Núñez A. et al.: A 5-formyltetrahydrofolate cycloligase paralog from all domains of life: comparative genomic and experimental evidence for a cryptic role in thiamin metabolism. - Funct. Integr. Genomic. 11: 467478, 2011.

Ruwe H., Kupsch C., Teubner M. et al.: The RNA-recognition motif in chloroplasts. - J. Plant Physiol. 168: 1361-1371, 2011.

Sarhadi E., Mahfoozi S., Hosseini S.A. et al.: Cold acclimation proteome analysis reveals close link between the up-regulation of low-temperature associated proteins and vernalization fulfillment. - J. Proteome Res. 9: 5658-5667, 2010.

Schuster G., Gruissem W.: Chloroplast mRNA 3' end processing requires a nuclear-encoded RNA-binding protein. - EMBO J. 10: 1493-1502, 1991.

Stern D., Goldschmidt-Clermont M., Hanson M.R.: Chloroplast RNA metabolism. - Annu. Rev. Plant Biol. 61: 125-155, 2010.
Sugita M., Ichinose M., Ide M. et al.: Architecture of the PPR gene family in the moss Physcomitrella patens. - RNA Biol. 10: 1439-1445, 2013.

Sun T., Germain A., Giloteaux L. et al.: An RNA recognition motif-containing protein is required for plastid RNA editing in Arabidopsis and maize. - P. Natl. Acad. Sci. USA 110: E1169E1178, 2013.

Teubner M., Fuß J., Kühn K. et al.: The RNA recognition motif protein CP33A is a global ligand of chloroplast mRNAs and is essential for plastid biogenesis and plant development. - Plant J. 89: 472-485, 2017.

Tillich M., Hardel S.L., Kupsch C. et al.: Chloroplast ribonucleoprotein $\mathrm{CP} 31 \mathrm{~A}$ is required for editing and stability of specific chloroplast mRNAs. - P. Natl. Acad. Sci. USA 106: 6002-6007, 2009.

Tillich M., Beick S., Schmitz-Linneweber C.: Chloroplast RNAbinding proteins. Repair and regulation of chloroplast transcripts. - RNA Biol. 7: 172-178, 2010.

Wang S., Bai G., Wang S. et al.: Chloroplast RNA-binding protein RBD1 promotes chilling tolerance through 23S rRNA processing in Arabidopsis. - PLoS Genet. 12: e1006027, 2016. 